\title{
Non-adiabatic effects of superconductor silane under high pressure
}

\author{
Fan Wei ${ }^{1}$ Wang Jiang-Long ${ }^{2,1}$, Zou Liang-Jian ${ }^{1}$, and Zeng Zhi ${ }^{1}$ \\ ${ }^{1}$ Key Laboratory of Materials Physics, Institute of Solid State Physics, Hefei Institutes of Physical Sciences, \\ Chinese Academy of Sciences, 230031-Hefei, People's Republic of China \\ ${ }^{2}$ Department of Physics, Hebei University, 071002-Baoding, People's Republic of China
}

(Dated: November 5, 2018)

\begin{abstract}
Investigations of non-adiabatic effects by including vertex corrections in the standard Eliashberg theory show that high phonon frequency is unfavorable to superconductivity in regime of strong vertex correction. This means that it is hard to find high-transition-temperature superconductors in the compounds with light elements if the non-adiabatic effects are strong. The interplay interaction between non-adiabatic effect and Coulomb interaction makes the transition temperature of silane superconductor not so high as predicted by the standard Eliashberg theory.
\end{abstract}

PACS numbers: $74.20 . \mathrm{Fg}, 74.62 . \mathrm{Fj}, 74.70 . \mathrm{Ad}$

The recently found superconductor silane $\left(\mathrm{SiH}_{4}\right)$ at high-pressure [1] partially realizes the theoretical predictions of high-temperature superconductors of hydrogen dominant metallic alloy [2] and metallic hydrogen at high pressure [3]. However, the transition temperature $\left(T_{c}\right)$ is significantly lower than the values predicted in previously theoretical calculations [4 7]. What is the underlaying mechanism of the lower $T_{c}$ compared with the higher $T_{c}$ predicted in strong-coupling theory. With increasing pressure the electronic structures of silane will evolve from molecule-like energy-levels to electron-bands when gaseous silane transforms into crystalline silane. The effective band-widths of conducting electrons of metallic silane are narrow so that the non-adiabatic effects will be prominent. It's very desirable study the influences of non-adiabatic effects on $T_{c}$.

The non-adiabatic effects are partially included in the vertex corrections in the theory of electron-phonon interaction. The Eliashberg theory [8-10] combined with the vertex corrections had been widely studied by perturbation method [1] 13]. We have performed a full parameter-space search based on Eliashberg theory with and without vertex corrections [13, 14]. In this paper, we study the influences of the vertex corrections on $T_{c}$ of superconductor silane at high pressure using reliable Eliashberg functions $\alpha^{2} F(\omega)$ obtained from the calculations of linear-response theory [4]. Our results indicate that the vertex correction can efficiently suppress $T_{c}$ approaching to the values found in experiments. Additionally, we find that high phonon frequency is unfavorable to high- $T_{c}$ if the strong vertex corrections are included. This means that it is hard to realize high- $T_{c}$ superconductors in silane and other hydrogen-rich materials.

We have generalized the equation of energy gap in Ref.[11] by including the Coulomb interaction [13]. The calculations of vertex corrections are greatly simplified under isotropic approximation. The electron-phonon interactions are included in the vertex corrections only by

*Electronic address: fan@theory.issp.ac.cn the functions $\lambda_{n}$ defined as $\lambda_{n}=2 \int_{0}^{\infty} d \nu \alpha^{2} F(\nu) \nu /\left(\nu^{2}+\right.$ $\left.\omega_{n}^{2}\right)$. When temperature is very close to $T_{c}$, the energy-gap equation including the leading vertex correction from electron-phonon interaction is written as $\sum_{n^{\prime}=-\infty}^{+\infty} K_{n n^{\prime}} \Delta_{n^{\prime}} /\left|\omega_{n^{\prime}}\right|=0$ with the kernel matrix

$$
K_{n n^{\prime}}=\left[\lambda_{n-n^{\prime}} B_{n n^{\prime}}-\mu^{*}+C_{n n^{\prime}}\right] a_{n^{\prime}}-\delta_{n n^{\prime}} H_{n^{\prime}}
$$

where the definitions of $H_{n}, A_{n n^{\prime}}, B_{n n^{\prime}}, C_{n n^{\prime}}$ and $a_{n}$ are presented in Ref.[11, 13]. In order to calculate $T_{c}$, the matrix $K_{n n^{\prime}}$ is symmetrized with the same manner as in Ref. 10]. The $E_{B}$ is the effective band-width and the Coulomb pseudo-potential $\mu^{*}$ is defined as $\mu^{*}=$ $\mu_{0} /\left(1+\mu_{0} \ln \left(E_{B} / \Omega_{0}\right)\right)$, where $\mu_{0}=N(0) U, N(0)$ the density of state at Fermi energy $E_{F}$, $\mathrm{U}$ the Coulomb interaction between electrons and $\Omega_{0}$ characteristic energy of typical phonon correlated to superconductivity. Generally, the ratio $\Omega_{0} / E_{B}$ takes as the parameter to measure vertex correction and the larger $\Omega_{0} / E_{B}$ is corresponding to the stronger vertex correction. In this context, the vertex corrections are controlled by $E_{B}$, the smaller $E_{B}$ (or larger $\Omega_{0} / E_{B}$ ) for stronger vertex corrections. With development of method of electronic-structure calculation in solid materials, the parameters of electronphonon interaction can be calculated using density functional theory combined with perturbing linear-response theory [15, 16]. The Elaishberg functions $\alpha^{2} F(\omega)$ obtained by linear response theory are easily merged in the formulae used in this context.

In the standard Eliashberg theory, $T_{c}$ will infinitely increase with phonon frequency or energy. It's widely expected that high-temperature superconductors should be found in compounds containing light elements such as hydrogen-rich material silane and metal hydrogen at high pressure. We will see that the non-adiabatic effects set bounds to infinitely increasing $T_{c}$. In order to obtain very general results, at first step, we apply the simple phonon spectrum and Eliashberg function $\alpha^{2} F(\omega)$ used in Ref. [9]. The Fig 1 shows how $T_{c}$ changes with $\Omega_{P}$ and $E_{B}$. We can see that for large band-width $E_{B}=5 \mathrm{eV}$ (weak vertex correction), $T_{C}$ increases monotonously with $\Omega_{P}$ just as in the standard Eliashberg theory. However for very strong vertex correction with small band-width $E_{B}=1 \mathrm{eV}, T_{c}$ is 


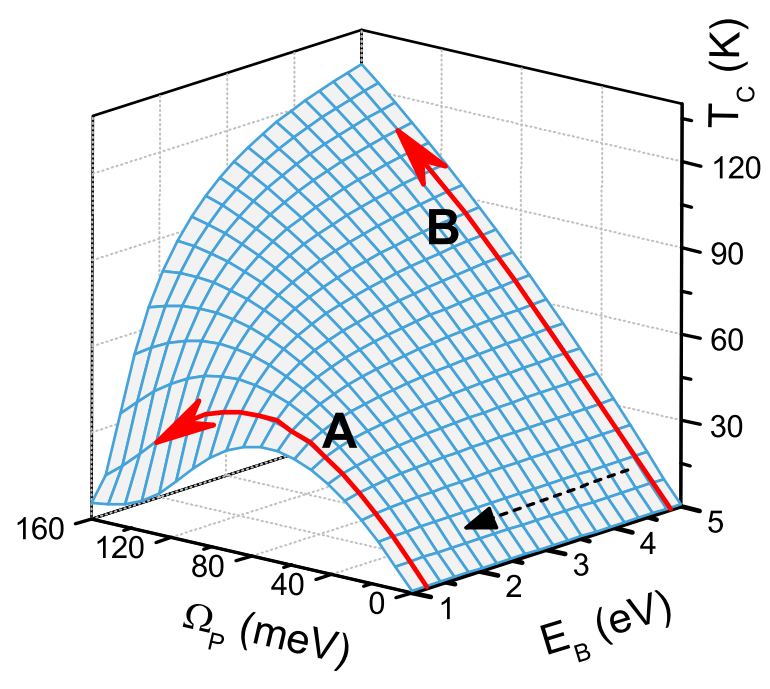

FIG. 1: The $T_{c}$ on $\Omega_{P}-E_{B}$ parameter plane. The two solid arrows $\mathbf{A}$ and $\mathbf{B}$ show the changes of $T_{c}$ with $\Omega_{P}$ at fixed $E_{B}$ (A) near $1 \mathrm{eV}$ and (B) $5 \mathrm{eV}$ respectively. The dashed arrow shows the direction enhanced the vertex corrections.

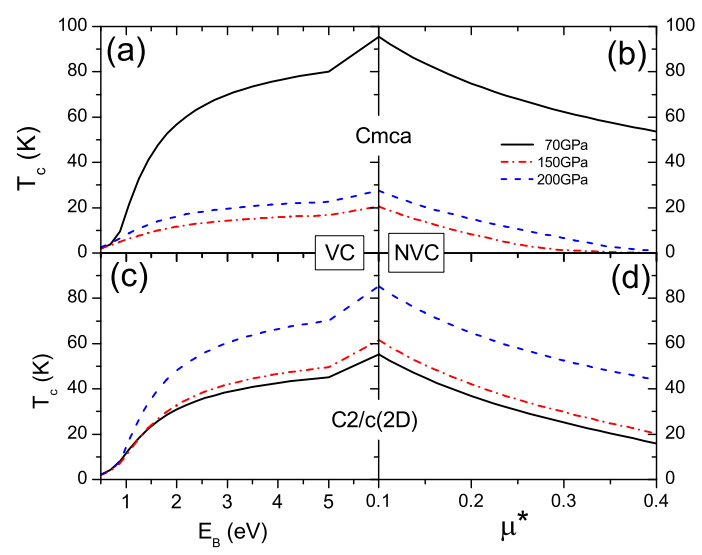

FIG. 2: The dependence of $T_{c}$ on $E_{B}$ and $\mu^{*}$, based on the Eliashberg functions $\alpha^{2} F(\omega)$ obtained from linear-response theory at different pressures for the $\mathrm{Cmca}$ structure $(\mathrm{a}, \mathrm{b})$ and $C 2 / c(2 \mathrm{D})$ structure $(\mathrm{c}, \mathrm{d})$ respectively [4]. The (a) and (c) include vertex corrections (VC), and there are no vertex corrections (NVC) in (b) and (d).

non-monotonously dependent on $\Omega_{P}$. When $\Omega_{P}$ is larger than a threshold value, $T_{c}$ will decrease with $\Omega_{P}$. This means that, if the vertex corrections are included, high phonon energy is unfavorable to superconductivity.

We preform the calculations of the vertex corrections of real superconducting material silane containing the lightest element: hydrogen. The lowest order vertex correction can significantly reduce $T_{c}$ when energy of phonon $\Omega_{P}$ is larger than $80 \mathrm{meV}$ shown in Fig 1 We adopt the Eliashberg functions that had been reported in Ref [4] in the calculations of linear-response theory. The structures of crystal silane at high-pressures haven't been completely defined. The metallic $P 6_{3}$ structure had been found in experiment [1]. The theoretical layered structure with $\mathrm{Cmca}$ space-group symmetry is more stable from 60-200 GPa [4], other stable structures at higher pressures had been already reported [17]. The hydrogen-rich superconductor silane at higher pressure has very high phonon energies coming from the vibrations of hydrogen atoms. The effective phonon energies $\langle\omega\rangle_{l n}$ for silane at high pressures distribute from $50 \mathrm{meV}$ to $75 \mathrm{meV}$ dependent on pressures. The parameters $\lambda$ of electron-phonon interaction are about $1.17,0.62,0.75$ for the Cmca structure and $0.84,0.87,1.1$ for the $C 2 / c(2 \mathrm{D})$ structure respectively when pressure increases from 70 GPa to $150 \mathrm{GPa}$ and to $200 \mathrm{GPa}$. The $T_{c}$ calculated using Allen-McMillan formula are distributed from $20 \mathrm{~K}$ to 80 $\mathrm{K}$ [4] that are greatly larger than experimental $T_{c}<17 \mathrm{~K}$.

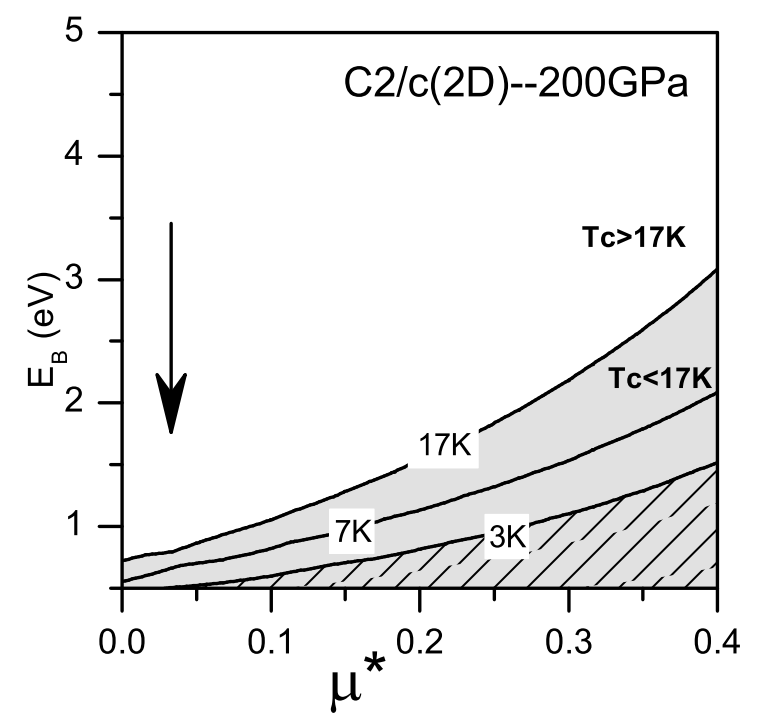

FIG. 3: The $T_{c}$ on $\mu^{*}-E_{B}$ plane using the Eliashberg functions $\alpha^{2} F(\omega)$ obtained from linear-response theory [4]. The structure has $C 2 / c(2 \mathrm{D})$ space group symmetry at pressure 200 GPa. The shade areas are regions with $T_{c}<17 \mathrm{~K}$. The arrow shows the directions of increasing the vertex correction.

As reported in Ref.[4], the pressure-dependence $T_{c}$ of the $C m c a$ structure is different from the $C 2 / c(2 \mathrm{D})$ structure. The $T_{c}$ of the $C m c a$ structure decreases when pressure increases from $70 \mathrm{GPa}$ to $150 \mathrm{GPa}$, and slightly increases with increasing pressure further to $200 \mathrm{GPa}$. However for the $C 2 / c(2 \mathrm{D})$ structure, $T_{c}$ monotonously increases with pressures from $70 \mathrm{GPa}$ to $200 \mathrm{GPa}$. We first present the results of the Cmca structure in detail shown in Fig 2(a,b). If the vertex corrections are not included and $\mu^{*}=0.1$, the values of $T_{c}$ are close to $20 \mathrm{~K}$ at pressures $150 \mathrm{GPa}$ and $200 \mathrm{GPa}$, which are smaller than those obtained from McMillan formula. So our results indicate that for very broad distributed phonon 
spectrum, the standard strong-coupling calculations are needed to accurately calculate $T_{c}$ beyond simple McMillan and Allen $T_{c}$ formulae.

At low pressure with larger inter-molecule distances, the crystalline silane is an insulator with narrow energy bands corresponding to discrete energy levels of a single molecule. The effective band-widths will increase with increasing pressures and overlaps of molecular orbits of different molecules. If the vertex corrections are included in our calculations with the effective band-width $E_{B}$ of conducting electrons from $0.5 \mathrm{eV}$ to $5 \mathrm{eV}$, the $T_{c}$ at 150 $\mathrm{GPa}$ and $200 \mathrm{GPa}$ are significantly reduced to the value smaller than $17 \mathrm{~K}$ shown in Fig2(a). The effective bandwidths obtained from the density functional calculations increase from very small values at low pressure to about 1.0-3.0 eV at 100-200 GPa for the $C 2 / c(2 \mathrm{D})$ and Cmca structures [4]. In another case, the effects of Coulomb interaction are more significant because the $T_{c}$ decreases to very small values with increasing $\mu^{*}$ at $150 \mathrm{GPa}$ and $200 \mathrm{GPa}$ shown in Fig2(b). If we want to know what is more important for the depression of $T_{c}$ we should know real value of $\mu^{*}$ at $150 \mathrm{GPa}$ and $200 \mathrm{GPa}$. At 70 $\mathrm{GPa}$, the small band-widths with $E_{B}<1 \mathrm{eV}$ guarantee the $T_{c}$ lower than $17 \mathrm{~K}$. We find that, if without vertex correction, even larger $\mu^{*}(=0.4)$ can not suppress $T_{c}$ to value smaller than $17 \mathrm{~K}$ at $70 \mathrm{GPa}$.

The effects of vertex correction of the $C 2 / c(2 \mathrm{D})$ structure in Fig2(c) are similar to the $\mathrm{Cmca}$ structure in Fig 2(a), however the influences of the Coulomb interaction in Fig_2(d) are different from the Cmca in Fig 2(b). The $C 2 / c(2 \mathrm{D})$ structure is less stable than the Cmca structure. The stronger electron-phonon interaction induces its structure more unstable at high pressures from $150 \mathrm{GPa}$ to $200 \mathrm{GPa}$. Theoretical values of $T_{c}$ increase with pressures. This is because the enhancements of $T_{c}$ induced by the increase of $\lambda$ are larger than the depressions of $T_{c}$ due to the vertex correction. For the $C 2 / c(2 \mathrm{D})$ structure, the Coulomb interaction depresses $T_{c}$ to values which are still larger than $17 \mathrm{~K}$ shown in Fig2(b). So Coulomb interaction individually can not explain why $T_{c}$ smaller than $17 \mathrm{~K}$ in high-pressure experiments. The effective band-widths $E_{B}$ of conducting electrons in the $C 2 / c(2 \mathrm{D})$ structure are larger than $1 \mathrm{eV}$ at $150 \mathrm{GPa}$ and $200 \mathrm{GPa}$ [4] so that the vertex correction individually can not explain low $T_{c}$ at $100 \mathrm{GPa}$ and $200 \mathrm{GPa}$ as well as shown in Fig 2(c). The Fig 3 illustrates the $T_{c}$ for the $C 2 / c(2 \mathrm{D})$ structure on $\mu^{*}-E_{B}$ plane at pressure $200 \mathrm{GPa}$. The shading region in the figure shows the effective region with parameters $\mu^{*}$ and $E_{B}$ that can explain the $T_{C}$ smaller than $17 \mathrm{~K}$, which is only small area of parameter-space with $\mu^{*}>0.1$ and $E_{B}<2.0 \mathrm{eV}$. Especially, the hatched region shows the parameter-space to explain why experimental $T_{c}$ is smaller $3 \mathrm{~K}$ at $200 \mathrm{GPa}$. So our results indicate that the interplay of vertex correction and Coulomb interaction can explain the low $T_{c}$ of the $\mathrm{C} 2 / \mathrm{c}(2 \mathrm{D})$ structure. Our calculations can not fully explain pressure-dependent $T_{c}$ in Ref [1], especially, the higher $T_{c}=17 \mathrm{~K}$ near 100-120 GPa because of the lack of information of structural changes.

In summary, we study the effects of the vertex correction on $T_{c}$ for superconductor of silane at high pressure. Our results indicate that the non-adiabatic effects are the barrier to prevent $T_{c}$ from increasing infinitely with phonon frequency. This means that it is hard to realize high $T_{c}$ to home temperature in high pressure richhydrogen materials. Our results also show that the interplay interaction between Coulomb interaction and vertex correction is essentially to explain the larger differences between theory and experiments.

The author (Fan W) thanks Dr. Li Yan-Ling for the helpful discussions. The work preforms in the Center for Computational Science of CASHIPS and is supported by Knowledge Innovation Project of Chinese Academy of Science.
[1] Eremets M I, Medvedev I A, Tse J S and Yao Y 2008 Science 3191506

[2] Ashcroft N W 2004 Phys. Rev. Lett. 92187002

[3] Ashcroft N W 1968 Phys. Rev. Lett. 211748

[4] Chen X J, Wang J L, Struzhkin V V, Mao H K, Hemley R J and Lin H Q 2008 Phys. Rev. Lett. 101077002 and supplemental materials

[5] Feng J, Grochala W, Jaroń T, Hoffmann R, Bergara A and Ashcroft N W 2006 Phys. Rev. Lett. 96017006

[6] Pickard C J and Needs R J 2006 Phys. Rev. Lett. 97 045504

[7] Yao Y, Tse J S, Ma Y and Tanaka K 2007 Eur. Phys. Lett. 7837003

[8] Éliashberg G M 1960 Soviet. Phys. JETP, 11696

[9] Scalapino D J, Schrieffer J R and Wilkins J W 1966 Phys.
Rev. 148263

[10] Allen P B and Dynes R C 1975 Phys. Rev. B 12905

[11] Kostur V N and Mitrović B 1994 Phys. Rev. B 5012774

[12] Grimaldi C, Pietronero L and Strässler S 1995 Phys. Rev. B 5210530

[13] Fan W 2009 Physica C 469177

[14] Fan W 2008 Chinese Phys. Lett. 252217

[15] Savrasov S Y and Savrasov D Y 1996 Physical Review B 5416487

[16] Baroni S, de Gironcoli S, Dal Corso A and Giannozzi P 2001 Rev. Mod. Phys. 73515

[17] Martinez-Canales M, Oganov A R, Ma Y M, Yan Y, Lyakhov A O and Bergara A 2009 Phys. Rev. Lett. 102 087005 